\title{
Tra visualità e visualizzazione Ai confini della «scienza dolce delle immagini» di W.J.T. Mitchell: una lettura*
}

\author{
Valentina Manchia \\ Politecnico di Milano, Dipartimento di Design; CROSS \\ valentina.manchia@polimi.it
}

\begin{abstract}
In a semiotic perspective, in dialogue with a science of images, J.W.T. Mitchell's Image Science (2015) appears interesting not only for the theoretical contributions here collected but for the argumentative structure bringing them together, that the author himself does not hesitate to propose as a metapicture of his system of thought. This essay therefore focuses both on the figurative (and figural) modalities of Mitchell's argumentative strategies and on the theoretical possibilities that an open reflection on interdisciplinary boundaries can open in different directions.
\end{abstract}

\section{Keywords}

Visual Culture Studies; Semiotics; Visual Semiotics; Image Theory; Verbo-Visual

\section{Contents}

1. Mitchell in forma di figura: una premessa semiotica

2. La chiave inglese come metapicture della scienza delle immagini e figura di confine 3. Tra scienze dure e scienze molli: confini e direzioni di ricerca tra visualità e visualizzazione

Bibliografia

* Questo saggio è la versione rivista, estesa e aggiornata di un primo contributo già pubblicato su Alfabeta2 il 2 dicembre 2018. 
Valentina Manchia | Tra visualità e visualizzazione

\section{Mitchell in forma di figura. Una premessa semiotica}

In un saggio sul frontespizio dei Principi di Scienza Nuova di Giambattista Vico (1737), Omar Calabrese dipana, con dovizia di dettagli, quella «sorta di tragitto dello sguardo che mette gli oggetti figurativi in connessione gli uni con gli altri» (Calabrese 1999: 327) e porta la «dipintura» del frontespizio a farsi carico dell'ossatura dell'argomentazione dei Principi e a darle corpo, sotto forma di figura.

L'idea di un frontespizio che, come in Vico e più in generale nella tradizione dei frontespizi figurati, «serve al leggitore per concepire l'idea di quest'opera avanti di leggere», quindi di una rappresentazione diagrammatica capace di restituire, sotto spoglie figurative, i rapporti di forza e le relazioni tra i concetti portanti dell'argomentazione, ci sembra utile anche qui, per leggere attraverso Mitchell.

La nostra idea, infatti, è che Scienza delle immagini non sia interessante solo per i contenuti teorici che mette insieme (molti dei quali già editi), ma per il modo in cui si occupa di tesserli insieme, e dunque per la figura dell'argomentazione che ne risulta. Il libro, infatti, dalla scelta dell'immagine di copertina alla struttura del volume, si occupa di testualizzare un momento teorico molto specifico: lo sguardo al presente, e allo stesso tempo dal proprio passato e dagli albori dei visual culture studies fino al presente, di uno degli autori chiave nell'ambito della visualità contemporanea.

L'esito di questa operazione di testualizzazione del proprio pensiero è la produzione di una figura al contempo esibita e nascosta, come nella Lettera rubata di Poe: quella della chiave inglese di Allan Sekula come figura del raccordo, della messa a sistema di prospettive teoriche differenti.

Il tentativo di queste pagine è dunque non solo quello di ripercorrere il pensiero di Mitchell in Scienza delle immagini ma anche quello di portare alla luce il pensiero diagrammatico interno ai saggi che lo costituiscono e che la struttura stessa del libro proietta.

In una prospettiva semiotica - e al contempo di scienza delle immagini - ci interesseranno dunque tanto le modalità figurative (e figurali) delle strategie argomentative di Mitchell quanto le possibilità teoriche che una riflessione aperta sui confini interdisciplinari può aprire in diverse direzioni teoriche.

\section{La chiave inglese come metapicture della scienza delle im- magini e figura di confine}

Campeggia sulla copertina di Scienza delle immagini. Iconologia, cultura visuale ed estetica dei media (Johan \& Levi, 2018) di W.J.T. Mitchell la stessa fotografia già scelta per l'edizione americana della University of Chicago Press: la chiave inglese che è allo stesso tempo chiave di volta del progetto fotografico di cui fa parte (Fish Story, 1995), ed emblema del "realismo sociale" del suo autore (Allan Sekula, fotografo e teorico della fotografia).

Di certo la chiave abbandonata, ritrovata e immortalata da Sekula due anni dopo la chiusura per bancarotta di un cantiere navale, è parte di una serie che è monumentale documentazione, dal 1988 al 1995, del cambiamento del 


\section{Dcula \\ Occhio semiotico sui media | Semiotic eye on media}

Valentina Manchia | Tra visualità e visualizzazione

lavoro sui mari a seguito degli stravolgimenti della globalizzazione. E a rappresentare questo cambiamento - il radicale passaggio da un prima, florido e attivo, a un dopo, improduttivo e vuoto - è in questo scatto e in altri della serie la frizione tra l'immagine e quello che all'immagine sta intorno, in tutti i sensi. Quello che vediamo sembra portarsi sempre dietro la ruggine, o la polvere, dello sfondo su cui inevitabilmente si staglia.

Qui, sulla copertina di Scienza delle immagini, estrapolata dalla serie di Sekula e posta in un nuovo contesto a offrirci un emblema del libro ma come vedremo di Mitchell stesso, tra i padri fondatori di quel campo interdisciplinare di studi, sempre più vasto e sempre più sfrangiato, che ha nome di cultura visuale, quella chiave inglese non può che farci riflettere.

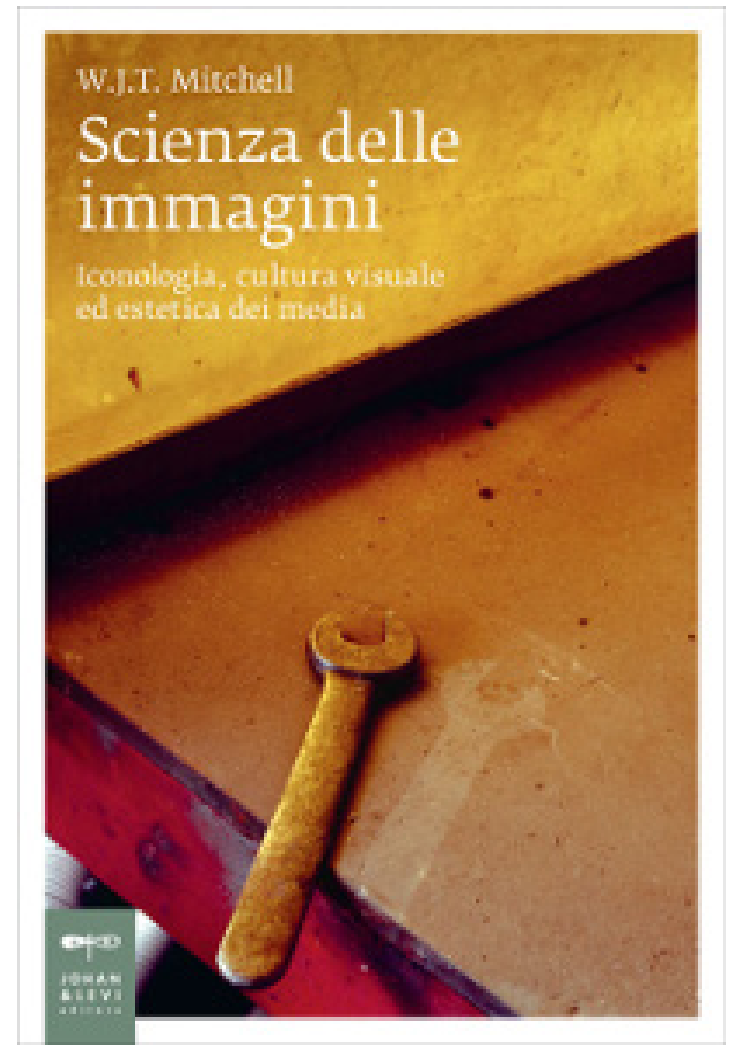

Figura 1. La copertina di Scienza delle immagini con lo scatto di Allan Sekula dalla serie Fish Story, 1995 (Welder's booth in bankrupt Todd Shipyard. Two years after closing. Los Angeles harbor. San Pedro, California)

È una sola immagine, dunque, quella che vediamo, o sono due immagini? Solo l'immagine, netta e precisa, sbalzata a colori saturi, di un oggetto solido e ben tangibile, o anche la traccia - fantasmatica - di quello stesso oggetto?

Proprio per questo doppio cortocircuito non poteva esserci copertina migliore per l'ultima raccolta di saggi di Mitchell, programmaticamente composta in un dittico, Figure e Sfondi, in cui la prima parte si assegna il compito di mettere insieme riflessioni che pertengono alla natura di quelle che chiamia- 
Valentina Manchia | Tra visualità e visualizzazione

mo immagini, per quanto diverse esse siano, e la seconda traccia un quadro - più quadri - sugli ambienti mediali e sulle condizioni di vita delle immagini.

Sfilano così, nella prima parte, contributi che fanno il punto sui concetti chiave di quella «iconologia critica» 0 «iconologia del presente» in cui Mitchell si è impegnato sin dai tempi di Iconology, la sua prima grande incursione in quel nuovo campo, dei visual culture studies, che avrebbe egli stesso attivamente concorso a costruire (Mitchell 1986).

Si tratta di concetti che sono poi filtrati, a volte anche sottotraccia, in molte altre riflessioni nell'ambito della cultura visuale: la nozione di pictorial turn, prima fra tutte, in parallelo all'iconic turn di Gottfried Boehm; la distinzione tra image e picture («le pictures sono la dimora in cui le images prendono residenza, i corpi in cui si incarna il loro spirito», Mitchell 2015: 77, tr. it.), con in controluce l'antropologia delle immagini di Hans Belting, ${ }^{1} \mathrm{o}$ il concetto, volutamente ibrido, di imagetext, immaginetesto, tutto attaccato e senza separazione tra i due, capace di accogliere in sé tanto quei testi che la semiotica chiamerebbe sincretici, perché fondati contemporaneamente su più linguaggi espressivi che si intersecano, ${ }^{2}$ quanto le immagini che si annidano anche tra le parole - per esempio dietro le metafore. O, ancora, il fortunato concetto di metapicture, immagine di immagini e in quanto tale dispositivo capace di offrire un nuovo angolo di riflessione - l'anatra-coniglio delle Ricerche filosofiche di Wittgenstein, per esempio, emblema dell'irriducibilità del punto di vista, a ricordarci che le immagini sono sempre immagini per qualcuno.

Già da questa breve rassegna è evidente come la riflessione di Mitchell si sia espansa, in mille rivoli, lungo il territorio via via sempre più esteso e variegato della cultura visuale - per una mappatura rimandiamo al puntuale e prezioso lavoro cartografico di Andrea Pinotti e Antonio Somaini (Pinotti-Somaini 2016) - ma soprattutto come abbia via via raccolto e convogliato le riflessioni e gli stimoli di altri autori e discipline che hanno fatto teoria delle immagini.

La selezione dei saggi per la seconda parte del dittico, Sfondi, chiarisce proprio la genesi di un punto di vista così plurale rintracciandone le origini nelle radici più profonde del pensiero dell'autore, arrivato all'iconologia da un percorso obliquo che egli stesso riassume, in un paragrafo che traccia l'intera parabola del suo lavoro:

Dal momento che io ero giunto allo studio della storia dell'arte dalla sfera della letteratura e della teoria letteraria, spronato da un interesse generale per la teoria da una parte e da un interesse specifico per l'arte composita del pittore-poeta William Blake dall'altra, mi sembrava sempre più ovvio che la materia effettiva del mio lavoro dovesse essere la relazione tra diversi media, forme artistiche, modalità sensoriali $\mathrm{e}$ codici di significazione, così come quella tra le discipline impegnate nel loro studio. (Mitchell 2015: 126, tr. it.)

1 Sull'antropologia delle immagini in Belting cfr. in particolare Belting 2001, e anche Belting 2005.

2 Sul sincretismo tra linguaggi espressivi differenti - e sulle semiotiche sincretiche come quelle semiotiche-oggetto che «costituiscono il loro piano di espressione [...] con elementi appartenenti a molte semiotiche eterogenee» cfr. Greimas-Courtès 1979-1986: 320, tr. it.). 


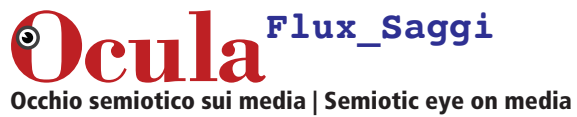

Valentina Manchia | Tra visualità e visualizzazione

Lo sguardo plurale di Mitchell si forma infatti da subito sull'«arte composita» di Blake, al tempo stesso poesia, pittura (illustrazione?) e grafica (calligrafia?), che diventa da subito l'evidenza di un'irriducibile commistione, quella tra testo e immagine - la madre, per l'autore di Iconology e dei successivi Picture Theory (1994), oltre che di What do pictures want? (2005), di tutte le commistioni tra media.

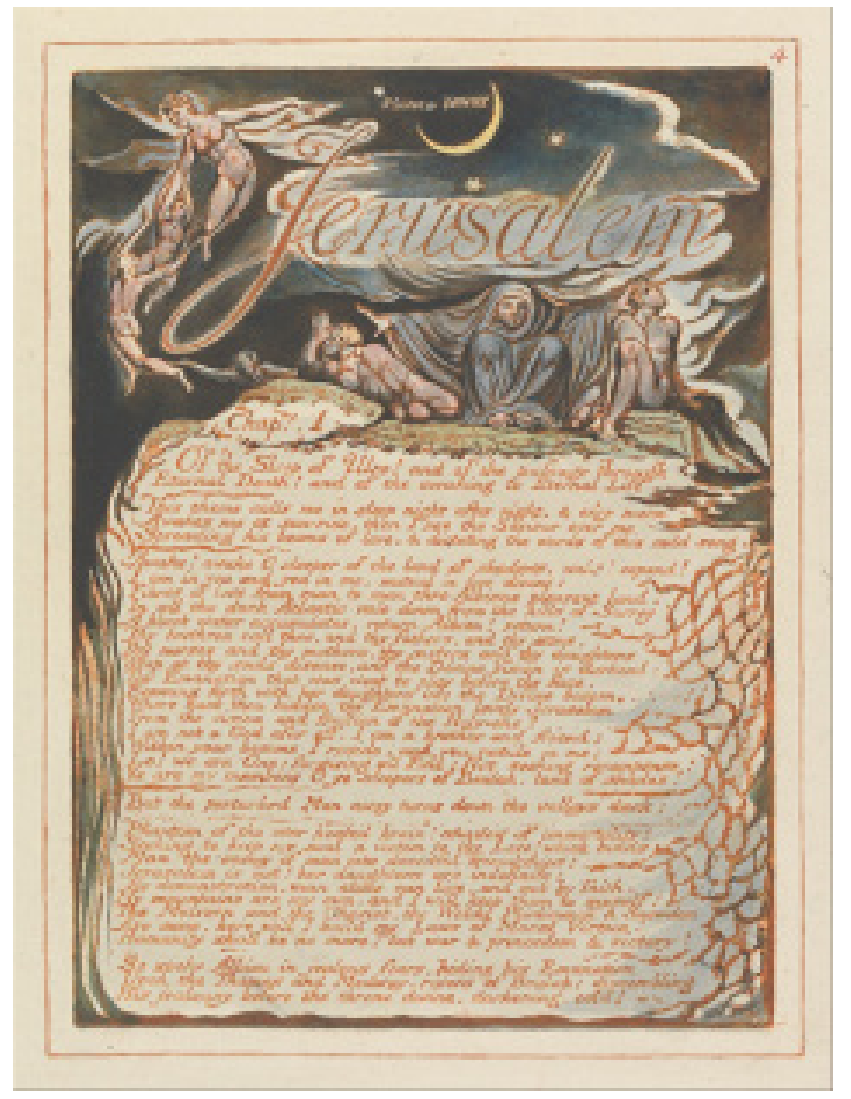

Figura 2. William Blake, Jerusalem, tavola 4, capitolo 1, 1804-1820.

«Dove comincia la scrittura? Dove comincia la pittura?» (Barthes 1984: 27 , tr. it.) era già la domanda di Roland Barthes, anch'egli sempre vigile nei confronti delle intersezioni tra forme e tra linguaggi, in particolare nell'interrogarsi sulla poesia figurata giapponese, ed è una domanda che molti altri studiosi sia all'interno del paradigma semiotico che nel campo interdisciplinare della cultura visuale si sono posti e continuano a porsi, riflettendo sulle molteplici intersezioni tra verbale e visivo. ${ }^{3}$

3 Facciamo riferimento, per esempio, a Schapiro (1996). Sempre all'interno del paradigma semiotico, ricordiamo in particolare Polacci 2010. Nell'ambito della cultura visuale James Elkins si è molto soffermato sia sulle intersezioni tra i due linguaggi che sui casi più estremi, quelli in cui si assiste a una sorta di inesorabile spostamento dalla scrittura all'immagine. Cfr. in particolare Elkins (1999). 


\section{Ocula \\ Occhio semiotico sui media | Semiotic eye on media}

Valentina Manchia | Tra visualità e visualizzazione

La stessa domanda sui confini tra i linguaggi, cambiando volta per volta oggetto, è quella che si ripete implicitamente Mitchell, ragionando sui media che sono l'ambiente naturale in cui vivono e proliferano le immagini. Ambienti o situazioni, più che dispositivi, sottolinea Mitchell in "Estetica dei media" (2013, in Mitchell 2015, pp. 119-130) e come tali capaci di fare da sfondo (ancora una volta, questa parola) a più modalità sensoriali, che spesso si innestano le une sulle altre.

Non esistono media puramente o esclusivamente visivi, né compartimenti stagni nella linea d'evoluzione temporale dei media. Opera invece un "processo continuo di ri-mediazione di forme più antiche» (seguendo Bolter-Grusin 1999), che rilegge continuamente forme e linguaggi, propone e ripropone le forme mediali del passato in nuove forme, e modella combinazioni diverse degli elementi estetici dei media, sempre in coerenza con «l'universo sensibile che abitiamo» (Mitchell 2015: 127, tr. it.).

Anche per questo sfilano, nei saggi raccolti in questo libro, la fotografia e l'architettura, la street art e la scultura, il terrorismo come guerra alle immagini e la clonazione come eccesso di immagine - temi interconnessi tra loro, al cuore del volutamente ambiguo Cloning Terror, che mette faccia a faccia il terrore della clonazione e la riproduzione incontrollata del Terrore (Mitchell 2011).

Scienza delle immagini riconnette concetti e riflessioni di saggi ormai classici e già noti ai lettori anche in traduzione italiana (in particolare i saggi ora in Mitchell 2017) a nuovi contributi, innestando al tessuto già consolidato da saggi come "Pictorial Turn" (1992) e da "Che cosa vogliono davvero le immagini?" (1996) riflessioni più recenti, come il già citato "Estetica dei media" (2013), tema quanto mai urgente nell'agenda della visual culture che discute di digital turn.

In questa prospettiva, in cui si dipanano i fili delle riflessioni precedenti per intrecciarle a un nuovo sguardo sul presente, è proprio Mitchell stesso, nella coda che conclude la silloge, a introdurre l'immagine dell'immagine di Sekula e a offrirla al lettore come una vera e propria metapicture del discorso che lega insieme i saggi della raccolta:

La fotografia cattura una doppia apparizione, una cosa reale e la sua impressione, quest'ultima rivelata da un leggero spostamento dell'oggetto che espone la sua traccia o immagine postuma. È una "stampa a contatto" che esemplifica il doppio carattere indessicale e iconico dell'immagine fotografica stessa, che produce significato attraverso la sua somiglianza formale e il suo status di traccia materiale simile a un'impronta. [...] Dunque, nonostante la sua collocazione nel mondo completamente artificiale di un cantiere navale moderno, essa ci ricorda che l'immagine è un fenomeno che attraversa i confini tra natura e cultura, tra i processi fisico-chimici di natura non umana e le creazioni dell'uomo. (Mitchell 2015: 227-228, tr. it.)

Presenza contemporanea di due apparizioni, quella di una cosa reale, la chiave inglese, e quella della sua evanescente impronta, la fotografia di Sekula è dunque deliberatamente lì per fare da segno - e come tale proponiamo di leggerla qui, in queste pagine -, ovvero per indicare la via e ricordarci le molteplici nature delle immagini. 


\section{Ocula \\ Occhio semiotico sui media | Semiotic eye on media}

Valentina Manchia | Tra visualità e visualizzazione

Il doppio profilo della chiave inglese, che scopriamo solo grazie al suo spostamento (non sappiamo quanto fortuito o quanto opera di Sekula, ma in fondo non è questo che ci interessa), allude direttamente, all'interno della cornice teorica di Mitchell, all'apparire di ogni image «come figura su uno sfondo, in un sistema annidato in un ambiente» (Mitchell 2015: 227, tr. it.), e alla complessità di ogni apparire che è all'incrocio tra la natura di ogni specifico visibile e le sue condizioni di vita. È dunque prima di tutto «allegoria grafica della relazione tra figura e sfondo, tra oggetto e spazio» (ibidem) ma è anche, secondariamente, rappresentazione della struttura portante dell'argomentazione, quasi un diagramma filosofico (per dirla con Fabbri 2015) del pensiero di Mitchell.

Da un'altra prospettiva, che lo stesso Mitchell introduce e somma alle precedenti in via metaforica, parlando della «tecnoscienza dolce delle immagini» come di «una sorta di chiave inglese per aggiustare le connessioni tra scienze dure e scienze molli» (Mitchell 2015: 228, tr. it), la chiave inglese di Sekula è lì per introdurre, sin dalla copertina, il tema più profondo delle riflessioni che Mitchell compie in Image Science, intorno e a margine del suo lavoro precedente sulle immagini, tema che corre sotterraneo attraverso tutti i saggi: quello del confine e della sua continua messa alla prova.

A partire da qui, più in senso figurativo (e figurale) che in senso metaforico, la chiave inglese abbandonata, a riposo dall'esercizio delle sue funzioni sul banco di un saldatore ormai in bancarotta («Welder's booth in bankrupt Todd Shipyard. Two years after closing», recita la didascalia), rimanda anche alle possibilità di connessione tra domini diversi ma comunque sovrapponibili, e in potenza solidali tra loro: le discipline delle immagini, da una parte, nel vasto dominio dei visual culture studies, ma soprattutto le scienze dure e le scienze umane, a loro modo alle prese con le immagini e forse, suggerisce Mitchell, sollecitate a riflettere su una saldatura possibile tra sguardi differenti. ${ }^{4}$

\section{Tra scienze dure e scienze molli: confini e direzioni di ricerca tra visualità e visualizzazione}

«Fenomeni che attraversano i confini» sono infatti le immagini, oggetti materiali e tangibili ma allo stesso tempo molto più di questo, capaci di migrare, trasformarsi e circolare liberamente, attraverso lo spazio e il tempo - come la dialettica tra image e picture ricorda e come, prima ancora, testimoniano Aby Warburg e il suo progetto di Atlante.

Ed è al centro delle attenzioni di Mitchell anche il confine tra discipline, che stimola frizioni (quando non attriti) tra iconologia e storia dell'arte da una parte e semiotica, sociologia dei media, e cultural studies dall'altra - confine già sfidato da chi, come e prima di Mitchell, ha provato ad allargare le ma-

4 Intendiamo qui, sulla scia del Dictionnaire (Greimas-Courtés 1986, voce Figura), il figurale come base fondante del figurativo, a prescindere dalle singole realizzazioni figurative sulla superficie del discorso. Una possibilità per sfuggire alla proliferazione di metafore visive per la nuova scienza delle immagini (dalla chiave inglese al guanto da boxe, cfr. Mitchell 2015: 223-229, tr. it.) e allo stesso tempo per comprenderle meglio, come operatori di un contatto (e di una presa) più fine sui confini delle cose. 
Valentina Manchia | Tra visualità e visualizzazione

glie dell'iconologia e della storia dell'arte e a prolungarne lo sguardo al di là dell'opera d'arte canonica. Pensiamo, in particolare, oltre al già citato Belting, a Alpers (1983) e al suo interesse non per l'arte olandese ma per la cultura visuale olandese, e a Elkins (1999) e al suo interesse nell'allargare le maglie di un "dominio delle immagini" che non può più contenere solo le art images ma deve invece aprirsi alle non-art images.

Tuttavia, all'origine della decisione di Mitchell di aver messo insieme, a dialogare tra di loro, questi saggi e non altri sta un'altra riflessione su un altro confine, quello più importante: quello tra scienze dure ed esatte e scienze "molli", ovvero tra le immagini come fondamento della conoscenza scientifica e le immagini come oggetto di indagine per le scienze umane.

Nella consapevolezza che né le prime né le seconde possono fare a meno delle immagini, la scienza dolce che Mitchell immagina in dialogo tra i due approcci proclama l'esigenza di guardare alle immagini - e a tutte le immagini, senza distinzioni - in modo scientifico, esatto, quantitativo, e qualitativo, denso, insieme. Senza dimenticarsi di noi che le guardiamo, prestando anzi «la stessa attenzione all'osservatore e all'osservato, al soggetto e all'oggetto», una cura che, per esempio, accanto ai territori percorsi dalla cultura visuale, il paradigma semiotico si premura da tempo di prodigare, attraverso lo sguardo sulle immagini proposto, primi tra tutti, da Louis Marin (pensiamo, in particolare, a Marin 1989 e Marin 1994) e rilanciato da Omar Calabrese e dalla sua scuola. ${ }^{5}$

L'ultimo confine da indagare, allora, corre proprio tra (e dentro) le immagini, tra quelle che - semplificando molto - ci ostiniamo ad ascrivere al regime dell'autentico, come la fotografia analogica, e quelle che tacciamo di manipolazione, come le immagini digitali; tra le arti che consideriamo puramente visive, frutto di procedure più o meno standard di rappresentazione, $\mathrm{e}$ quelle che invece riconosciamo come ibride, composite, frutto di una sintesi che oltrepassa in vari modi la visualità standard.

Siamo proprio sicuri, provoca Mitchell, che certe immagini scientifiche capaci di farci vedere l'invisibile non abbiano nulla a che vedere con la manipolazione, nel momento in cui ci offrono un «guadagno di realtà»? Perché sembriamo preoccuparci più dell'aderenza delle immagini digitali al loro oggetto e non delle nuove possibilità di controllo sulla produzione e sulla circolazione delle immagini che il digitale offre? E ancora: perché non parlare anche di migrazione - e di clandestinità - delle immagini, oltre che di circolazione?

Sono temi capitali, che è interessante ritrovare nello sguardo di un Mitchell che ripercorre i suoi propri passi, sin dai tempi di Iconology, ben calcandone l'impronta, e che percorrono appunto, sotto altre forme, molte altre riflessioni: per intercettarne solo alcune, quella di Françoise Bastide, biologa e semiologa, vicina a Latour ma allieva di Greimas, e delle sue riflessioni sulliconografia delle immagini scientifiche, oltre che delle loro strategie comunicative; ${ }^{6}$

5 Sul pensiero di Omar Calabrese sulla semiotica dell'immagine ci riferiamo in particolare a Calabrese (1985), ma cfr. anche Lancioni (2013) e Corrain-Lancioni (2012), per gli sviluppi successive interni alla sua scuola. (1985).

6 Cfr. per esempio i saggi raccolti in Bastide (2001), in particolare Bastide 


\section{Ocula \\ Occhio semiotico sui media | Semiotic eye on media}

Valentina Manchia | Tra visualità e visualizzazione

quella di Peter Galison, e dei suoi studi sull'oggettività scientifica come fatto di visione; ${ }^{7}$ quella di Lev Manovich, sul modo in cui i software contribuiscono a dare forma alla nostra esperienza nel mondo digitale, ${ }^{8}$ e quella di Victor Stoichita, sull'alterità nella visualità e sulle molteplici «immagini dell'altro» (Stoichita 2014).

Ora più che mai, più o meno implicitamente - ora che il livello delle immagini che ci sommerge sale sempre più velocemente giorno dopo giorno - siamo consapevoli che nessun occhio è innocente, come ammoniva saggiamente Ernst Gombrich, e che quello che vediamo in realtà contribuiamo a costruirlo, ma occorre anche prendere coscienza della complessità della visione e dei domini delle immagini:

Se dobbiamo avere una scienza delle immagini, allora, il primo passo è quello di liberarla dalla tirannia dell'occhio fisico e corporeo, inteso in senso letterale, e riconoscere che le immagini [...] attraversano differenti domini: ci sono immagini mentali, matematiche e verbali così come ce ne sono di pittoriche e visive. (Mitchell 2015: 40, tr. it.)

Bisogna, ovvero, esplicitare il passo successivo, per la «scienza dolce delle immagini» ma più in generale per le scienze che guardano alle immagini $\mathrm{e}$ attraverso le immagini: non soltanto la visione è socialmente costruita e situata, non soltanto la costruzione del nostro vivere sociale è sempre più visuale, ma è il nostro stesso modo di pensare e di misurare il mondo che passa sempre più inevitabilmente attraverso le immagini, intrecciando in modo sempre più complesso l'approccio puramente quantitativo delle scienze dure a quello denso delle scienze umane, per esempio per guardare all'ambiente digitale.

"How to see one billion images", per esempio, è il titolo dell'introduzione di Lev Manovich al suo nuovo libro, Cultural Analytics (2020), in cui si propone di illustrare le dinamiche del suo metodo di direct visualization, alla ricerca di una restituzione immediata, quantitativa ma aperta all'analisi qualitativa, del visibile. E sempre più forte, nel campo dei big data, è l'esigenza di provare a mettere a punto procedure di visualizzazione che non siano soltanto ingegneristiche ma che sappiano cogliere le sfumature di una complessità che è percepita come densa (si sente sempre più spesso parlare, infatti, di thick data, di dati di un'altra natura, "estratti" attraverso procedure etnografiche, quindi di osservazione e di ascolto). ${ }^{9}$

È da questa prospettiva che l'ibrido di Mitchell di una scienza che sappia essere anche dolce - e che a chi scrive ricorda il Paolo Fabbri che invitava a mantenere un dialogo tra scienze umane e scienze disumane - può essere utile per guardare a una visualità sempre più complessa come quella contemporanea.

7 Cfr. in particolare Daston-Galison (2007).

8 Cfr. in particolare Manovich (2013) e (2017).

9 Sui thick data cfr. per esempio, Wang (2016). Il riferimento di Wang, nel coniare l'espressione thick data, è a Clifford Geertz (1973) e alla «thick description» dell'antropologia e dell'etnografia. 


\section{Dcula ${ }^{\text {Flux__aggi }}$ \\ Occhio semiotico sui media | Semiotic eye on media}

Valentina Manchia | Tra visualità e visualizzazione

\section{Bibliografia}

Alpers, Svetlana

1983 The Art of Describing: Dutch Art in the Seventeenth Century, Chicago, University of Chicago Press (tr. it. Arte del descrivere. Scienza e pittura nel Seicento olandese, Torino, Boringhieri, 1984).

Barthes, Roland

1970 L'empire des signes, Genève-Paris, Skira-Flammarion (tr. it. L’impero dei segni, Torino, Einaudi, 1984).

Bastide, Françoise

1985 "Iconographie des textes scientifiques: principes d'analyse", Culture Technique, 14 (tr. it. "Iconografia dei testi scientifici. Principi d'analisi", in Una notte con Saturno. Scritti semiotici sul discorso scientifico, a cura di B. Latour, P. Fabbri, Roma, Meltemi, 2001, pp. 167-213).

2001 Una notte con Saturno. Scritti semiotici sul discorso scientifico, a cura di B. Latour, P. Fabbri, Roma, Meltemi.

Belting, Hans

2001 Bild-Anthropologie. Entwürfe für eine Bildwissenschaft, München, Fink (tr. it. Antropologia delle immagini, Roma, Carocci, 2011).

2005 "Image, medium, body. A new approach to iconology", Critical Inquiry, 31, pp. 302-319 (tr. it. "Immagine, medium, corpo: un nuovo approccio all'iconologia", in Teorie dell'immagine. Il dibattito contemporaneo, a cura di Andrea Pinotti e Antonio Somaini, Milano, Raffaello Cortina, 2009, pp. 73-98).

Bolter, Jay. David, Grusin, Richard

1999 Remediation. Understanding new media, Cambridge, MIT Press (tr. it. Remediation. Competizione e integrazione tra media vecchi e nuovi, Milano, Guerini, 2003).

Calabrese, Omar

1985 La macchina della pittura, Bari, Laterza (nuova ed. Firenze, La casa Usher, 2012).

1999 "La memoria geroglifica. Riflessioni semiotiche sul frontespizio dei Principi di Scienza Nuova di Giambattista Vico", in Eloquio del senso, a cura di Pierluigi Basso e Lucia Corrain, Milano, Costa \& Nolan, 1999, pp. 324-336.

Corrain, Lucia; Lancioni, Tarcisio

2012 "Geometrie del senso. Ripensare la semiotica dell'immagine a partire da $\mathrm{La}$ macchina della pittura di Omar Calabrese”, E/C. Rivista dell'Associazione Italiana di Studi Semiotici.

Daston, Lorraine; Galison, Peter

2007 Objectivity, New York, Zone Books.

Elkins, James

1999 The Domain of Images, Ithaca, NY, Cornell University Press.

Fabbri, Paolo

2015 "Diagrammi in filosofia: G. Deleuze e la semiotica "pura", in Immagini che fanno segno. Modi e pratiche di rappresentazione diagrammatica nelle informational images, a cura di Valentina Manchia, Carte Semiotiche. Rivista Internazionale di Semiotica e Teoria dell'Immagine, pp. 27-35. 


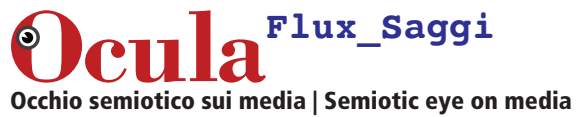

Valentina Manchia | Tra visualità e visualizzazione

Geertz, Clifford

1973 “Thick Description: Toward an Interpretive Theory of Culture", in Id., The Interpretation of Cultures: Selected Essays, New York, Basic Books, pp. 3-30.

Greimas, Algirdas J., Courtés, Jacques

1979-1986 Sémiotique. Dictionnaire raisonné de la théorie du langage, I-II, Paris, Hachette (tr. it. Semiotica. Dizionario ragionato della teoria del linguaggio, Usher, Firenze, 1986, ora anche tr. parz. in Semiotica. Dizionario ragionato della teoria del linguaggio, Milano, Bruno Mondadori, 2007).

Lancioni, Tarcisio

2013 "Immagini in prospettiva. Forme e figure dell'enunciazione visiva", in Semiotica delle soggettività. Per Omar, a cura di Massimo Leone e Isabella Pezzini, Aracne, Roma, pp. 281-288.

Manovich, Lev

2013 Software Takes Command, New York, Bloomsbury Academic.

2017 Instagram and Contemporary Image, http://manovich.net/index.php/ projects/instagram-and-contemporary-image

2020 Cultural Analytics, Cambridge, Mass.-London, MIT Press.

Marin, Louis

1994 De la représentation, Paris, Le Seuil/Gallimard (tr. it. Della rappresentazione, a cura di L. Corrain, Milano, Mimesis, 2014).

1989 Opacité de la peinture, Essais sur la représentation au Quattrocento, Paris, Usher (tr. it. Opacità della pittura. Saggi sulla rappresentazione nel Quattrocento, Firenze, La casa Usher, 2012).

Mitchell, W.J.T.

1986 Iconology. Image, Text, Ideology, Chicago, The University of Chicago Press.

1992 "The Pictorial Turn", Artforum, 30, pp. 89-94 (tr. it. "Pictorial Turn”, in Id., Pictorial turn. Saggi di cultura visuale, a cura di Michele Cometa e Valeria Cammarata, Milano, Raffaello Cortina, 2017, pp. 79-106).

1994 Picture theory. Essays on verbal and visual representation, Chicago, University of Chicago Press.

1996 "What do Pictures Really Want?”, October, 77, pp. 71-82 (tr. it. "Che cosa vogliono davvero le immagini?”, in Id., Pictorial turn. Saggi di cultura visuale, a cura di Michele Cometa e Valeria Cammarata, Milano, Raffaello Cortina, 2017, pp. 107-124).

2005 What Do Pictures Want? The Lives and Loves of Images, Chicago, University of Chicago Press.

2011 Cloning terror. The war of images, 9/11 to the present, Chicago, The University of Chicago Press (tr. it. Cloning terror. La guerra delle immagini dall'11 settembre a oggi, Lucca, La casa Usher, 2012).

2013 «Media Aesthetics», foreword to Thinking Media Aesthetics

Media Studies, Film Studies and the Arts, edited By Liv Hausken, New York, Peter Lang, pp. 15-18 (tr. it. "Estetica dei media", in Scienza delle immagini. Iconologia, cultura visuale ed estetica dei media, Monza, Johan \& Levi, 2019, pp. 119-130).

2015 Image Science. Iconology, Visual Culture, and Media Aesthetics, Chicago, The University of Chicago Press (tr. it. Scienza delle immagini. Iconologia, cultura visuale ed estetica dei media, Monza, Johan \& Levi, 2019).

2017 Pictorial turn. Saggi di cultura visuale, a cura di Michele Cometa e Valeria Cammarata, Milano, Raffaello Cortina.

Pinotti, Andrea; Somaini, Antonio

2016 Cultura visuale. Immagini, sguardi, media, dispositivi, Torino, Einaudi. 


\section{Ocula \\ Flux_Saggi \\ Occhio semiotico sui media | Semiotic eye on media}

Valentina Manchia | Tra visualità e visualizzazione

Polacci, Francesca

2010 "Dispositivi sincretici: per una semiotica verbo-visiva", in Dario Tomasello, Francesca Polacci, Bisogno furioso di liberare le parole. Tra verbale e visivo: percorsi analitici delle Tavole parolibere futuriste, Firenze, Le Lettere, 2010, pp. 83-185.

Sekula, Allan

1995 Fish story, Düsseldorf, Richter.

Schapiro, Meyer

1996 "Script in Pictures: Semiotics of Visual Language" in Words, Script and Pictures. Semiotics of Visual Language, New York, Braziller (tr. it. "Scritte in pitture: la semiotica del linguaggio visivo" in Id., Per una semiotica del linguaggio visivo, Roma, Meltemi, 2002, pp. 192-236).

Stoichita, Victor I.

2014 L'image de l'Autre. Noirs, Juifs, Musulmans et «Gitans» dans l'art occidental des Temps Modernes, Paris, Hazan, 2014 (tr. it. L’immagine dell'Altro. Neri, giudei, musulmani e gitani nella pittura occidentale dell'Età moderna, Firenze, La Casa Usher, 2019).

Wang, Tricia

2016 "Why Big Data Needs Thick Data”, Etnography Matters, <https://medium. com/ethnography-matters/why-big-data-needs-thick-data-b4b3e75e $3 \mathrm{~d} 7 \#$. xhtmw64pe>, 20 gennaio 2016.

Valentina Manchia Dottore di ricerca in Semiotica e Comunicazione simbolica presso l'Università di Siena, attualmente insegna al Politecnico di Milano, all'Alma Mater Studiorum - Università di Bologna, a ISIA Urbino e IAAD (Bologna e Torino), dove si occupa di semiotica, comunicazione e progettazione visiva, design e cultura visuale. Ha curato, per La Casa Usher, il numero di Carte Semiotiche dal titolo Immagini che fanno segno. Modi e pratiche di rappresentazione diagrammatica nelle informational images (2015). Suoi saggi e articoli sono apparsi, oltre che in pubblicazioni collegate a convegni nazionali e internazionali, su Lexia, E/C, Progetto grafico, Alfabeta2, Doppiozero. È membro del CROSS - Centro di Ricerca "Omar Calabrese" di Semiotica e Scienze dell'immagine. 